\title{
MedGA: A novel evolutionary method for image enhancement in medical imaging systems
}

Leonardo Rundo, email: leonardo.rundo@disco.unimib.it

University of Milano-Bicocca - Department of Informatics, Systems and Communication, 20126 Milano, Italy

Institute of Molecular Bioimaging and Physiology, Italian National Research Council, 90015 Cefalú (PA), Italy

Andrea Tangherloni, email: andrea.tangherloni@disco.unimib.it

University of Milano-Bicocca - Department of Informatics, Systems and Communication, 20126 Milano, Italy

Marco S. Nobile, email: email: marco.nobile@disco.unimib.it

University of Milano-Bicocca - Department of Informatics, Systems and Communication, 20126 Milano, Italy

SYSBIO.IT Centre of Systems Biology, 20126 Milano, Italy

Carmelo Militello, email: carmelo.militello@ibfm.cnr.it

Institute of Molecular Bioimaging and Physiology, Italian National Research Council, 90015

Cefalú (PA), Italy

Daniela Besozzi, email: daniela.besozzi@unimib.it

University of Milano-Bicocca - Department of Informatics, Systems and Communication, 20126 Milano, Italy

Giancarlo Mauri, email: mauri@disco.unimib.it

University of Milano-Bicocca - Department of Informatics, Systems and Communication, 20126 Milano, Italy

SYSBIO.IT Centre of Systems Biology, 20126 Milano, Italy

Paolo Cazzaniga* email: paolo.cazzaniga@unibg.it, +39035 2052933

University of Bergamo - Department of Human and Social Sciences, Piazzale Sant'Agostino

2, 24129 Bergamo, Italy

SYSBIO.IT Centre of Systems Biology, 20126 Milano, Italy

* Corresponding author 


\title{
MedGA: A novel evolutionary method for image enhancement in medical imaging systems
}

\author{
Leonardo Rundo ${ }^{\mathrm{a}, \mathrm{b}, * *}$, Andrea Tangherloni ${ }^{\mathrm{a}, * *}$, Marco S. Nobile ${ }^{\mathrm{a}, \mathrm{c}}$, \\ Carmelo Militello $^{\mathrm{b}}$, Daniela Besozzi ${ }^{\mathrm{a}}$, Giancarlo Mauri ${ }^{\mathrm{a}, \mathrm{c}}$, Paolo Cazzaniga ${ }^{\mathrm{d}, \mathrm{c}, *}$ \\ ${ }^{a}$ University of Milano-Bicocca - Department of Informatics, Systems and Communication, \\ 20126 Milano, Italy \\ ${ }^{b}$ Institute of Molecular Bioimaging and Physiology, Italian National Research Council, \\ 90015 Cefalú (PA), Italy \\ ${ }^{c}$ SYSBIO.IT Centre of Systems Biology, 20126 Milano, Italy \\ ${ }^{d}$ University of Bergamo - Department of Human and Social Sciences, 24129 Bergamo, Italy
}

\begin{abstract}
Medical imaging systems often require the application of image enhancement techniques to help physicians in anomaly/abnormality detection and diagnosis, as well as to improve the quality of images that undergo automated image processing. In this work we introduce MedGA, a novel image enhancement method based on Genetic Algorithms that is able to improve the appearance and the visual quality of images characterized by a bimodal gray level intensity histogram, by strengthening their two underlying sub-distributions. MedGA can be exploited as a pre-processing step for the enhancement of images with a nearly bimodal histogram distribution, to improve the results achieved by downstream image processing techniques. As a case study, we use MedGA as a clinical expert system for contrast-enhanced Magnetic Resonance image analysis, considering Magnetic Resonance guided Focused Ultrasound Surgery for uterine fibroids. The performances of MedGA are quantitatively evaluated by means of various image enhancement metrics, and compared against the conventional state-of-the-art image enhancement techniques, namely, histogram equalization, bi-histogram equalization, encoding and decoding Gamma transformations, and sigmoid transformations. We show that MedGA considerably
\end{abstract}

\footnotetext{
${ }^{*}$ Corresponding author: paolo.cazzaniga@unibg.it

**These authors contributed equally
}

Preprint submitted to Expert Systems with Applications

October 25, 2018 
outperforms the other approaches in terms of signal and perceived image quality, while preserving the input mean brightness. MedGA may have a significant impact in real healthcare environments, representing an intelligent solution for Clinical Decision Support Systems in radiology practice for image enhancement, to visually assist physicians during their interactive decision-making tasks, as well as for the improvement of downstream automated processing pipelines in clinically useful measurements.

Keywords: Medical imaging systems, Image enhancement, Genetic

Algorithms, Magnetic resonance imaging, Bimodal image histogram, Uterine fibroids

\section{Introduction}

Nowadays, medical imaging systems play a key role in the clinical workflow, thanks to their capability of representing anatomical and physiological features that are otherwise inaccessible to inspection, thus proposing accurate imag-

5 ing biomarkers and clinically useful information (Rueckert et al., 2016; Lambin et al. 2017). Medical images are considerably different from the pictures usually analyzed in Pattern Recognition and Computer Vision, as regards the appearance of the depicted objects as well as the information conveyed by the pixels. As a matter of fact, medical imaging techniques exploit several different principles to measure spatial distributions of physical attributes of the human body, allowing us to better understand complex or rare diseases (Toennies, 2017). The effectiveness of such techniques can be reduced by a plethora of phenomena, such as noise and partial volume effects (Toennies, 2017), which might affect the measurement processes involved in imaging and data acquisition devices.

15 In addition, computer-aided medical image acquisition procedures generally include reconstruction methods (producing two, three, or even four dimensional imaging data), which could cause the appearance of artifacts. Image contrast and details might also be impaired by the procedures used in medical imaging, as well as by the physiological nature of the body part under investigation. 
Medical images actually convey an amount of information-mainly related to high image resolution and high pixel depth - that could overwhelm the human vision capabilities in distinguishing among dozens of gray levels (Ortiz et al. 2013). Improving the appearance - and the visual quality - of medical images is therefore essential to provide physicians with valuable information that would not be immediately observable in the original image, assisting them in anomaly detection, diagnosis and treatment. This kind of diagnosis includes two basic processes: image observation (visual perception), and diagnostic interpretation (cognition) (Krupinski, 2010). Errors occurring in these diagnostic and therapeutic decision-making processes may have a significant impact on patient care, most notably possible misdiagnoses. In this context, image enhancement techniques aim at realizing a specific improvement in the quality of a given medical image. The enhanced image is expected to better reveal certain features, compared to their original appearance (de Araujo et al., 2014). In particular, these methods could have a significant clinical impact when the dynamic range of the actual pictorial content is not commensurable with the range of the displayed data (i.e., monitor luminance response), as well as when the input image is characterized either by a high level of noise or by a low contrast (Paranjape, 2009, Gonzalez \& Woods, 2002). This also applies to specialized computer screens for diagnostic reporting.

$40 \quad$ Although the majority of the enhancement techniques are typically applied to generate improved images for a human observer, others are exploited as a preprocessing step to provide enhanced images to further algorithms for computerassisted analyses (Paranjape, 2009). The first category includes techniques devoted to remove noise, enhance contrast and sharpen the details. The second category, partially overlapped with the former one, includes additional techniques such as edge detection and object segmentation for automated processing (Rangayyan, 2009). It was shown that a high-contrast medical image could lead to a better interpretation of the different adjacent tissues in the imaged body part (Chen et al. 2015). Accordingly, the resulting enhanced imagein terms of signal intensities of different tissues - can facilitate the automated 
segmentation, feature extraction, and classification of these tissues.

In the clinical routine, Contrast-Enhanced (CE) Magnetic Resonance Imaging (MRI) is a diagnostic technique that enables a more precise assessment of the imaged tissues after the administration of a Gadolinium-based contrast medium 55 in patients (Sourbron \& Buckley, 2013). MRI is currently the most prominent modality to obtain soft-tissue imaging (Brown et al., 2014), especially in oncology, since it provides significant improvements - in terms of image contrast and resolution - between lesion and healthy tissue (Metcalfe et al., 2013). For these reasons, MRI is considered more suitable than Computed Tomography in determining the extent of cancer infiltration. Furthermore, the excellent MRI soft-tissue contrast has led to an increasing role of this modality in target volume delineation for therapy applications, such as image-guided surgery and radiotherapy treatment, and for patients follow-up (i.e., staging and assessing tumor response) (Evans, 2008). However, MRI data are affected by acquisition noise 65 (Styner et al., 2000) and are also prone to imaging artifacts, related to magnetic susceptibility and large intensity inhomogeneities of the static magnetic field (i.e., streaking or shadowing artifacts (Bellon et al., 1986)), especially using high magnetic field strengths. These aspects make MR image enhancement a challenging task aiming at improving the results of automatic segmentation methods. The existing image enhancement approaches generally attempt to improve the contrast level of the whole image and do not address the issues related to overlapped gray level intensities; as a consequence, neither the region contour sharpness nor the image thresholding results can be improved. In the case of threshold-based image segmentation with two classes (i.e., foreground and background) (Muangkote et al., 2017), the input image is assumed to have a bimodal distribution of the histogram bins (Xue \& Zhang, 2012). Thus, an appropriate image enhancement method that yields medical images with a sharper bimodal distribution is required. However, determining the best pre-processing of an image - able to preserve the structural information of the image, while enhancso ing the underlying bimodal distribution - is a complex task on a multi-modal fitness landscape that demands the use of global optimization approaches. 
This paper presents a novel image enhancement technique based on Genetic Algorithms (GAs) (Holland, 1992), called MedGA, specifically aimed at strengthening the sub-distributions in medical images with an underlying bimodal histogram of the gray level intensities. Among the existing soft computing methods for global optimization, GAs represent the most suitable technique for this application, because of the discrete structure of the candidate solutions and the intrinsic combinatorial structure of the problem under investigation.

In this work, we apply MedGA to a clinical context involving CE MR image analysis, i.e., Magnetic Resonance guided Focused Ultrasound Surgery (MRgFUS) for uterine fibroids. The performances of MedGA are quantitatively evaluated by means of the most relevant image enhancement metrics, and compared against the conventional state-of-the-art image enhancement techniques, namely, histogram equalization, bi-histogram equalization, encoding and decoding Gamma transformations, and three instances of sigmoid transformation. Considering the possible clinical applications, MedGA is able to improve the visual perception of a Region of Interest (ROI) in MRI data with an underlying bimodal intensity distribution. In addition, MedGA can be used as an intelligent pre-processing step, in red any pipeline defined to realize an efficient threshold-based image segmentation with two classes (i.e., binarization), applied to expert systems working on MRI data. Indeed, image thresholding approaches performed on CE MR image regions could considerably benefit from input data pre-processed by MedGA.

The main contributions of MedGA in the context of expert and intelligent clinical systems can be briefly outlined as follows. MedGA acts as an expert system by playing a two-fold role: $(i)$ image enhancement to visually assist physicians during their interactive decision-making tasks, and ( $i i)$ improvement of the results in downstream automated processing pipelines for clinically useful measurements. The rationale behind the development of MedGA is the need of an intelligent model that is well-suited to effectively enhance medical images with roughly bimodal histograms. To the best of our knowledge, MedGA is the first work that explicitly deals with the improvement of thresholding- 
based segmentation results (Xue \& Zhang, 2012). Therefore, our computational framework can be employed as an intelligent solution in Clinical Decision Support Systems (CDSSs). As a matter of fact, MedGA represents an interpretable computational model (Castelvecchi, 2016) that allows for the understandability of the results (i.e., the gray level histogram is readable by the user). The compelling issues related to the interpretability of Machine Learning and Computational Intelligence methods in medicine are fundamental for the adoption and the clinical feasibility of a novel CDSS (Cabitza et al., 2017). In addition, no user interaction is required thanks to a reliable calibration step for the parameter settings of the GA. The goal of this paper consists in showing that evolutionary computation methods can boost the state-of-the-art performance in medical image enhancement, thus fostering GAs as a new concrete support tool for the clinical practice. Such an expert system may have a significant impact in real healthcare environments.

This manuscript is organized as follows. Related literature works and a theoretical comparison with existing methods are outlined in Section 2, Section 3 describes the MedGA image enhancement method. The evaluation metrics and the MR images used to assess the performance of MedGA are described in Section 4 Section 5 presents the achieved experimental results, by extensively explaining parameter analysis of MedGA. Finally, discussions and conclusive remarks are reported in Sections 6 and 7 , respectively.

\section{Background}

\subsection{Related work}

Most of the existing enhancement techniques are empirical or heuristic methods - strongly related to a particular type of images - that generally aim at improving the contrast level of images degraded during the acquisition process (Chen et al. 2018). As a matter of fact, finding the best gray level mapping that adaptively enhances each different input image can be considered an optimization problem (Paulinas \& Ušinskas, 2007, Draa \& Bouaziz, 2014). 
Unfortunately, no unifying theory employing a standardized image quality measure is currently available to define a general criterion for image enhancement (Munteanu \& Rosa, 2004). In addition, in the case of medical imaging, techniques tailored on specific tasks are necessary to achieve a significant enhancement and, in general, interactive procedures involving considerable human effort are needed to obtain satisfactory results.

In order to achieve objective and reproducible measurements conveying clinically useful information, operator-dependence should be minimized by means of automated methods. Point-wise operations in the spatial (pixel) domain, representing the simplest form of image processing, are effective solutions since efficiency requirements have also to be met. In the case of image enhancement, they re-map each input gray level into a certain output gray level, according to a global transformation (Gonzalez \& Woods, 2002). Thus, such kind of techniques treat images as a whole, without considering specific features of different regions, or selectively distinguishing between a collection of contrast enhancement degrees or settings (Munteanu \& Rosa, 2004). Histogram Equalization (HE) is the most common global image enhancement technique, whose aim is to uniformly redistribute the input gray level values according to the cumulative density function of its histogram (Gonzalez \& Woods, 2002, Hall, 1974). Unfortunately, HE does not take into account the image mean intensity Chen \& Ramli 2003), which is subject to a significant change during the equalization process by invariably shifting the output mean brightness to the middle gray level, regardless of the mean gray level in the input image (Gan et al., 2014). Consequently, HE is not able to preserve the input mean brightness, possibly suffering from over-enhancement, and giving rise to artifacts such as the socalled washed-out effect (Chen \& Ramli, 2003). This global transformation method applies contrast stretching just on gray levels with the highest frequencies, causing a significant contrast loss concerning the gray levels characterized 170 by low frequencies in the input histogram (Kim, 1997). Bi-Histogram Equalization (Bi-HE), which is a refined version of the traditional HE, was proposed to overcome the limitations related to input mean brightness preservation, mainly 
caused by histogram flattening (Kim, 1997). Firstly, Bi-HE splits the original histogram into two sub-histograms according to the global mean of the original image; afterwards, the sub-histograms are independently processed by applying the standard HE method to each of them.

The complexity of the enhancement criteria to be met (i.e., the effective contrast stretching combined with image detail preserving) leads to the application of global search meta-heuristics that allow for coping with several constraints, which are not generally tractable by means of traditional exhaustive computational approaches (Paulinas \& Ušinskas, 2007; Munteanu \& Rosa, 2004 Ortiz et al. 2013). Evolutionary methods have been widely adopted in the image enhancement domain to find the optimal enhancement kernel (Munteanu \& Rosa 2004), sequence of filters (Kohmura \& Wakahara, 2006), or input-output mapping transformation (Saitoh, 1999, Carbonaro \& Zingaretti, 1999). Recently, (Hashemi et al. 2010) proposed a GA-based method that efficiently encodes the histogram by means of the non-zero intensity levels, by employing genetic operators that directly process images to increase the visible details and contrast of low illumination regions, especially in the case of high dynamic ranges. The authors argued that this method yields "natural-looking" images, considering the visual appearance.

Regarding other evolutionary computation approaches, Genetic Programming (GP) (Koza, 1992) was shown to be a powerful framework to select and combine existing algorithms in the most suitable way. Differently to GAs, GP evolves a population of functions, or more generally, computer programs to solve a computational task. The solutions in the computer program space can be represented as trees, lines of code, expressions in prefix or postfix notations as well as strings of variable length (Castelli et al., 2014). For instance, (Bianco et al., 2017) tackled the video change detection problem (among the frames 200 of video streams) by combining existing algorithms via different GP solutions exploiting several fusion schemes. The fitness function was composed of different performance measures regarding change detection evaluation. For what concerns the application of GP in image enhancement, (Poli \& Cagnoni, 1997) 
proposed an approach to yield optimally pseudo-colored images for visualization

It is worth noting that all works mentioned in Section 2.1 are focused on consumer electronics or medical applications, to obtain more "visually pleasant" images by mainly increasing the contrast of the whole image. On the contrary, 
the main key novelty of MedGA consists in better revealing the two underlying

damental role for threshold-based segmentation approaches, since they strongly rely on the assumption that the bimodal histogram under investigation is composed of two nearly Gaussian distributions with almost equal size and variance (Xue \& Zhang, 2012).

MedGA also differs from GP-based approaches whose generated solutions might have a large size (Castelli et al., 2014), even when the GP model is implemented efficiently, thus representing a limitation that could significantly impair the readability and interpretability of the final outcome. Moreover, MedGA does not require any user interaction step, differently to (Poli \& Cagnoni, 1997) 


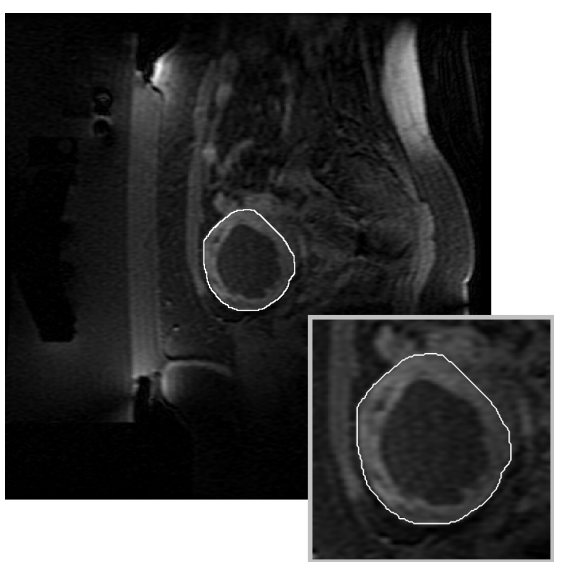

(a)

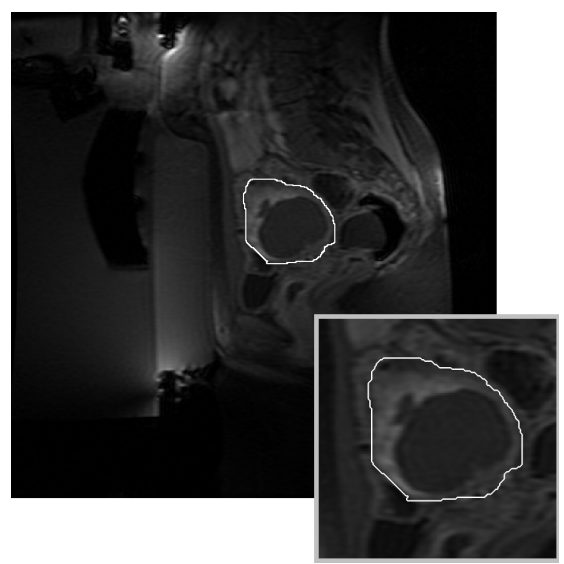

(b)

Figure 1: Examples of MR images. The ROI bounding region (i.e., the delineated uterus region), which includes the actual ROI (i.e., the uterine fibroid region), is highlighted with a white contour. The image regions including the ROIs, zoomed at the bottom right of each sub-figure, are characterized by nearly bimodal histograms.

where the user, being directly involved in the tournament selection, controls the evolution of simple programs that enhance and integrate multiple gray-scale images into a single pseudo-color image.

\section{MedGA: an intelligent method based on Genetic Algorithms for medical image enhancement}

MedGA is a global enhancement technique able to improve the details of medical images characterized by an underlying bimodal histogram of gray levels. Given a medical image wherein a ROI needs to be enhanced to achieve further analyses, MedGA aims at improving the ROI quality to facilitate the classification among different neighboring tissues, in order to support both the interpretation tasks by experienced radiologists and automated image analysis approaches.

The image enhancement carried out by MedGA focuses on the pixels within a sub-region of the input MRI, called ROI bounding region, including the ROI 
itself. To be more precise, starting from an MR image (Fig. 1), a bounding initialized by sampling $n$ integer values from the discrete uniform distribution in $\mathcal{L}^{\prime}{ }_{i n}$. The $n$ values are then sorted in ascending order so that the intensity levels $C_{i}(j)$ (with $j=1, \ldots, n$ ) codified by the individual can be mapped to 
At each iteration of MedGA, a number of individuals properly selected from the current population are inserted into intermediate populations, and modified by means of crossover and mutation operators. Note that, at each iteration, each individual $C_{i}$ belonging to the current population codifies for an ordered ${ }_{335}$ set $\mathcal{L}_{\text {out }, i}=\left[C_{i}(1), \ldots, C_{i}(n)\right]=\left[l_{\text {out }, i}^{(\min )}, \ldots, l_{\text {out }, i}^{(\max )}\right]$, which represents a modified gray level distribution of $\mathcal{L}^{\prime}{ }_{i n}$. In order to simplify the notation, in what follows we do not explicitly express that, at each iteration, each individual $C_{i}$ is (possibly) represented by a different circular array corresponding to $\mathcal{L}_{\text {out }}$.

For the selection of individuals, MedGA exploits a tournament strategy for

the intensity levels of the input MR image (i.e., the gray level frequencies of the input MR image are assigned to the corresponding intensity levels of the individual). During the initialization of the individuals, if an integer value is sampled more than once then the frequency values of the input MR image, corresponding to these gray level intensities, are summed up and assigned to

The rationale of MedGA is to process the ordered set $\mathcal{L}^{\prime}$ in by modifying its gray levels using a sequence of genetic operators, to obtain a solution characterized by a stronger bimodal gray level distribution in an output gray-scale range $\mathcal{L}_{\text {out }}=\left[l_{\text {out }}^{(\min )}, \ldots, l_{\text {out }}^{(\max )}\right] \subset \mathbb{N}$, where any element of $\mathcal{L}_{\text {out }}$ is also an element of $\mathcal{L}^{\prime}{ }_{i n}$. In such a way, a direct mapping between the gray levels of the original image and the final one is defined, so that each gray level in the original histogram is replaced with the gray level value contained by the same position in the final best solution $C_{\text {best }} \in P$. MedGA realizes this global intensity transformation by improving the separation between $\mathcal{H}_{1, i}$ and $\mathcal{H}_{2, i}$, which represent the dark spectively. To this aim, MedGA exploits the optimal threshold $\theta_{o p t, i}$ adaptively selected using the Iterative Optimal Threshold Selection (IOTS) method (Ridler \& Calvard, 1978, Trussell, 1979). This procedure yields enhanced medical images that better reveal the bimodal intensity distribution in computer-assisted ROI extraction tasks.

hree main reasons: $(i)$ the selection pressure can be controlled by setting the 
tournament size $k$ (with $k \ll|P|$ ); (ii) the fitness evaluations are performed only on the $k$ individuals selected for the tournaments, and not on the whole population; (iii) this technique could be easily implemented on parallel architectures (Miller \& Goldberg, 1995).

A single point crossover operator is applied with a given probability $p_{c}$ to the individuals selected by the tournament strategy and belonging to the first intermediate population $P^{\prime}$. Namely, given two parent individuals $C_{m}, C_{f} \in P^{\prime}$ (for some $m, f=1, \ldots,|P|$ ), a crossover point is randomly selected from the circular arrays $\left[C_{m}(1), C_{m}(2), \ldots, C_{m}(n)\right]$ and $\left[C_{f}(1), C_{f}(2), \ldots, C_{f}(n)\right]$ encoding the individuals, and two offspring are generated by swapping $50 \%$ of the values between the two parents. The offspring are then inserted into a second intermediate population $P^{\prime \prime}$.

The mutation operator is applied with probability $p_{m}$ to each element $C_{i}(j) \in$ $C_{i}=\left[l_{\text {out }, i}^{(\min )}, \ldots, l_{\text {out }, i}^{(\max )}\right]$ of each individual belonging to $P^{\prime \prime}$, where $l_{\text {out }, i}^{(\min )}$ and $l_{\text {out }, i}^{(\max )}$ are the minimum and maximum non-zero gray levels encoded by $C_{i}$ during the current iteration, respectively. In particular, if the gray level intensity encoded in $C_{i}(j)$ is smaller than the optimal threshold $\theta_{\text {opt }, i}$ evaluated by IOTS at that iteration for the individual $C_{i}$, then an integer is randomly sampled from the uniform distribution in $\left[l_{\text {out }, i}^{(\min )}, \ldots, \theta_{\text {opt }, i}-1\right] \subset \mathbb{N}$ to update the value $360 C_{i}(j)$; otherwise, an integer is randomly sampled from the uniform distribution in $\left[\theta_{\text {opt }, i}, \ldots, l_{\text {out }, i}^{(\max )}\right] \subset \mathbb{N}$ to update the value $C_{i}(j)$.

Finally, to prevent the quality of the best solution from decreasing during the optimization, MedGA also exploits an elitism strategy, so that the best individual from the current population is copied into the next population without undergoing the genetic operators.

Fig. 2 illustrates the overall procedure of MedGA, by presenting the initialization phase as well as the flow diagram of the proposed GA for image enhancement. The final best solution shows the achieved result on MRI data characterized by a bimodal histogram, emphasizing the two underlying distributions for the subsequent image thresholding phase, according to the optimal adaptive threshold $\theta_{\text {opt }}$, computed on the final best solution $C_{\text {best }}$, by using the 
simple IOTS algorithm (Ridler \& Calvard, 1978, Trussell, 1979).
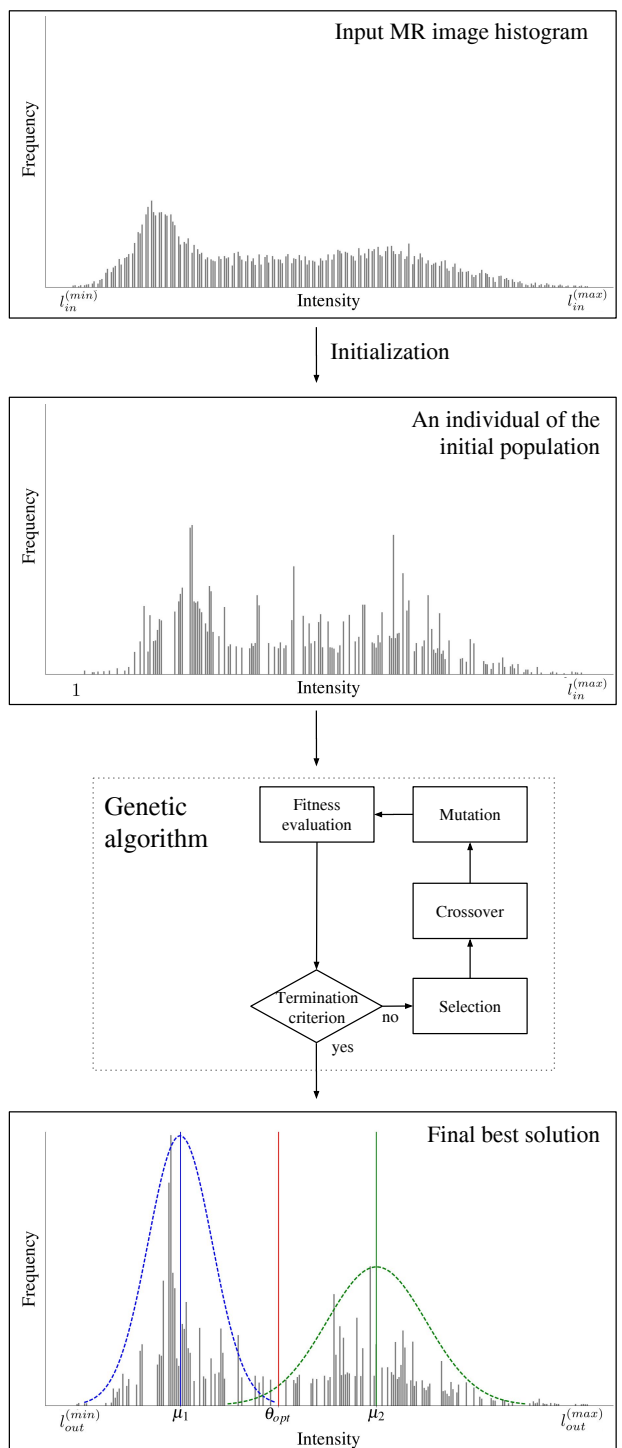

Figure 2: Workflow of MedGA: the individuals are initialized according to the characteristics of the input MR image, and processed by the GA. The final best solution of MedGA strengthens the two underlying distributions in the gray levels intensity characterized by mean values $\mu_{1}$ and $\mu_{2}$ and standard deviations $\sigma_{1}$ and $\sigma_{2}$, respectively. The two distributions are highlighted in the plot with blue and green dashed lines. 
To evaluate the quality of the individuals throughout the optimization, the fitness function has been conceived to obtain a bimodal histogram in the gray levels intensities, therefore facilitating further automated image processing phases. The fitness function $\mathcal{F}(\cdot)$ used by MedGA fosters individuals $C_{i}$ characterized by a histogram with two well separated normal distributions, having an equal distance from the optimal threshold $\theta_{o p t, i}$. To this purpose, at each iteration MedGA estimates, by using the efficient IOTS algorithm (Ridler \& Calvard, 1978, Trussell, 1979), the mean values $\mu_{1, i}$ and $\mu_{2, i}$ of the two components $\mathcal{H}_{1, i}$ and $\mathcal{H}_{2, i}$ of the histogram $\mathcal{H}_{i}$, encoded by each individual $C_{i}$, according to the current optimal threshold $\theta_{o p t, i}$. Afterwards, at each iteration the fitness function of every individual $C_{i}$ is calculated as follows:

$$
\begin{array}{ll}
\mathcal{F}\left(C_{i}\right) & =\tau_{1}+\tau_{2}+\tau_{3}, \quad \text { where: } \\
\tau_{1} & =\left|2 \cdot \theta_{o p t, i}-\mu_{1, i}-\mu_{2, i}\right|, \\
\tau_{2} & =\left|\omega_{1, i}-3 \sigma_{1, i}\right|, \\
\tau_{3} & =\left|\omega_{2, i}-3 \sigma_{2, i}\right| .
\end{array}
$$

The terms $\omega_{1, i}=\frac{1}{2}\left(\theta_{o p t, i}-\min _{j \in\{1, \ldots, n\}}\left\{C_{i}(j)\right\}\right)$ and $\omega_{2, i}=\frac{1}{2}\left(\max _{j \in\{1, \ldots, n\}}\left\{C_{i}(j)\right\}-\right.$ $\left.\theta_{\text {opt }, i}\right)$ correspond to the half width of $\mathcal{H}_{1, i}$ and $\mathcal{H}_{2, i}$, respectively, while $\sigma_{1, i}$ and $375 \sigma_{2, i}$ are the standard deviations of $\mathcal{H}_{1, i}$ and $\mathcal{H}_{2, i}$, respectively. The three terms of the fitness function $\mathcal{F}(\cdot)$ cooperate together to achieve the desired image enhancement: $\tau_{1}$ aims at maintaining the mean values $\mu_{1, i}$ and $\mu_{2, i}$ equidistant from the yielded optimal threshold $\theta_{o p t, i}$, while $\tau_{2}$ and $\tau_{3}$ are designed to force the sub-histograms $\mathcal{H}_{1, i}$ and $\mathcal{H}_{2, i}$ to approximate normal distributions.

We exploit the empirical property of normal distributions related to the coverage probability with respect the standard deviation. To be more precise, we consider the so-called $3-\sigma$ rule, which states that approximately $99.73 \%$ of the values lie within $3 \sigma$ according to: $\operatorname{Pr}(\mu-3 \sigma \leq X \leq \mu+3 \sigma) \approx 0.9973$, where $\mu, \sigma$ and $X$ represent the mean, the standard deviation and an observation from 385 a normally distributed random variable, respectively.

Examples of MR image enhancement results, achieved by MedGA on uterine fibroid, are shown in Fig. 3. MedGA enhances the input MR image by making 


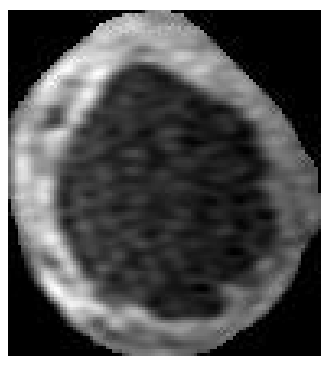

(a)

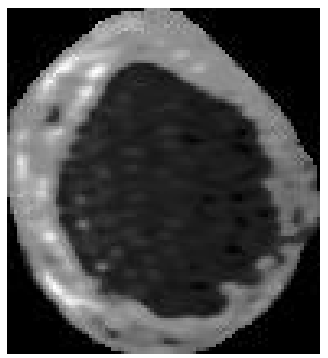

(c)

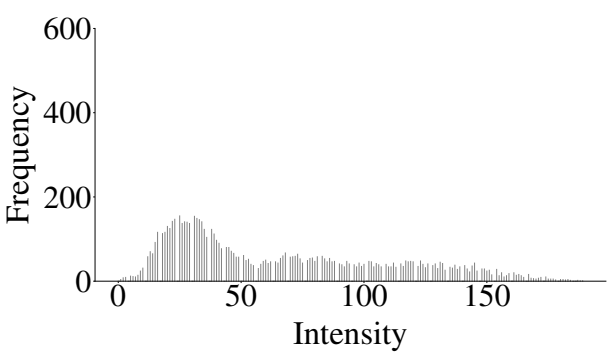

(b)

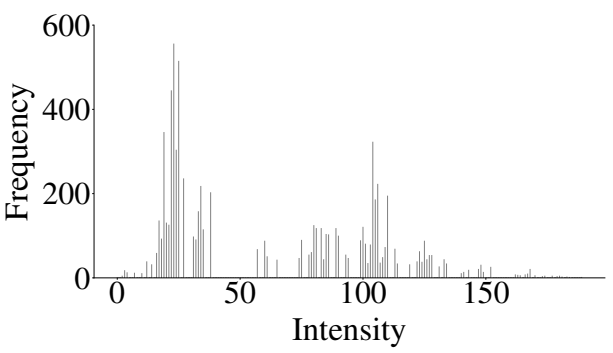

(d)

Figure 3: Enhanced image obtained by MedGA on uterine fibroids of patients who had undergone MRgFUS therapy: (a) input MR image normalized by using linear contrast stretching on the initial full range of the masked MR image; (c) output image after the application of MedGA. Plots (b) and (d) report the histograms of gray level intensities corresponding to the MR images in (a) and (c), respectively.

uterine fibroid regions more uniform and with strong edges in terms of both visual human perception and automated image processing. The histogram in Fig. $3 \mathrm{~d}$ proves that the output image is characterized by a more defined bimodal distribution compared to the initial image (Fig. 3p), which roughly tends to a trimodal gray level distribution.

It is worth noting that, in the case of further automated analysis, ROI pixel classification can be carried out by means of a basic threshold-based segmentation approach, since MR images enhanced with MedGA reveal a more precise separation between the two (possibly overlapping) sub-distributions in the histogram. Accordingly, MedGA allows for dealing with image histograms that do not meet the assumptions imposed by thresholding techniques, regarding bi- 
modal histograms composed of two nearly Gaussian distributions with almost equal size and variance (Xue \& Zhang, 2012). Indeed, MedGA enhances the image thresholding results on MRI data, as shown in Fig. 2, where the final best solution improves the underlying bimodal nature of the input histogram.

A sequential and a parallel version of MedGA have been implemented. The sequential version has been entirely developed using the Python programming language (version 2.7.12), while the parallel version is based on a Master-Slave paradigm employing mpi4py, which provides bindings of the Message Passing Interface (MPI) specifications for Python to leverage High-Performance Computing (HPC) resources (Dalcín et al., 2005).

\section{Materials and evaluation metrics}

\subsection{MRI dataset}

Eighteen patients affected by symptomatic uterine fibroids, who underwent MRgFUS therapy, were taken into account. The total number of the examined fibroids was 29 represented on $163 \mathrm{CE}$ MR slices, since some patients presented a pathological scenario with multiple fibroids. The analyzed images were acquired using a Signa HDxt MRI scanner (General Electric Medical Systems, Milwaukee, WI, USA) at two different institutions. These MRI data were acquired after the MRgFUS treatment, executed with the ExAblate 2100 (Insightec Ltd., Carmel, Israel) HIFU equipment. The considered MRI series were scanned using the T1w "Fast Spoiled Gradient Echo + Fat Suppression + ${ }_{420}$ Contrast mean" (FSPGR $+\mathrm{FS}+\mathrm{C}$ ) protocol. This MRI protocol is usually employed for Non-Perfused Volume (NPV) assessment (Gorny et al., 2011), since ablated fibroids appear as hypo-intense areas due to low perfusion of the contrast medium. Sagittal MRI sections were processed, in compliance with the current clinical practice for therapy response assessment (Militello et al., 2014).

${ }_{425}$ MRI acquisition parameters were: Repetition Time (TR): 150-260 ms; Echo Time (TE): 1.392-1.544 ms; pixel depth: 16-bit; matrix size: $512 \times 512$ pixels; 
slice thickness: $5.0 \mathrm{~mm}$; slice spacing: $6.0 \mathrm{~mm}$; pixel spacing: 0.6641-0.7031 $\mathrm{mm}$.

As explained in Section 3, before applying MedGA image enhancement, the uterus region (i.e., ROI bounding region) must be delineated. This task can be accomplished manually by the user or automatically by means of computational methods to reduce operator-dependency, as previously proposed in Militello et al. 2015b). Afterwards, the pixels with values lower than the optimal threshold $\theta_{\text {opt }}$, computed by means of the efficient IOTS method (Ridler \& Calvard, 1978, Trussell, 1979), are segmented in the binarized MR image. In this clinical scenario, segmentation approaches must deal with NPV inhomogeneities, due to sonication spots during the MRgFUS treatment.

\subsection{Image enhancement evaluation metrics}

In this section, we recall the definition of the metrics typically used to evaluate image enhancement approaches, which will be exploited to assess the performance of MedGA. These metrics are essential to quantitatively evaluate the effects of image enhancement techniques, since measuring the "quality" of an image might be strongly subjective. In particular, we benefit here from the metrics considered in (Hashemi et al. 2010) to assess the capability of image enhancement approaches in improving contrast, details and human visual perception.

Let $I_{\text {orig }}$ and $I_{\text {enh }}$ be the original input image and the enhanced image, respectively, consisting in $M$ rows and $N$ columns. Considering that the range of gray levels of the output image $\mathcal{L}_{\text {out }}=\left[l_{\text {out }}^{(\min )}, \ldots, l_{\text {out }}^{(\max )}\right]$ could be different from the original range $\mathcal{L}_{i n}=\left[l_{\text {in }}^{(\min )}, \ldots, l_{\text {in }}^{(\max )}\right]$, as a first step before computing the metrics we remap the output pixel intensities (i.e., the gray levels) into the original range, as follows:

$$
\tilde{I}_{\text {enh }}(a, b)=\frac{\left(I_{\text {enh }}(a, b)-l_{\text {out }}^{(\min )}\right) \cdot\left(l_{\text {in }}^{(\max )}-l_{\text {in }}^{(\min )}\right)}{\left(l_{\text {out }}^{(\text {max })}-l_{\text {out }}^{(\min )}\right)}+l_{\text {in }}^{(\min )},
$$

where $a=1, \ldots, M$ and $b=1, \ldots, N$. Note that we actually consider the extended range $\mathcal{L}_{\text {in }}^{\prime}$ for $I_{\text {orig }}$, as explained in Section 3 . 
The Peak Signal-to-Noise Ratio (PSNR) denotes the ratio between the maximum possible intensity value of a signal and the distortion between the input and output images:

$$
\begin{aligned}
P S N R & =10 \cdot \log _{10}\left(\frac{\left(l_{\text {in }}^{(\max )}\right)^{2}}{M S E}\right) \\
& =20 \cdot \log _{10}\left(\frac{l_{\text {in }}^{(\max )}}{\sqrt{M S E}}\right)
\end{aligned}
$$

where $M S E=\frac{1}{M \times N} \sum_{a=1}^{M} \sum_{b=1}^{N}\left\|I_{\text {orig }}(a, b)-\tilde{I}_{e n h}(a, b)\right\|^{2}$ is the Mean Squared Error, which allows us to compare the pixel values of $I_{\text {orig }}$ to those of $\tilde{I}_{e n h}$.

Furthermore, the PSNR is usually expressed in terms of the logarithmic Decibel scale. With regard to our application, we employ only a limited portion of the full dynamic range of the 16-bit images (see Section 3); we thus use as the largest possible value the maximum intensity value present in the original image ${ }_{455}$ (i.e., $l_{\text {in }}^{(\max )}=\max \left\{\mathcal{L}_{i n}\right\}=\max \left\{\mathcal{L}^{\prime}{ }_{i n}\right\}$ ) instead of the maximum representable value in a 16 -bit image (i.e., $\left.2^{16}-1=65,535\right)$.

Munteanu \& Rosa (2004) stated that good contrast and enhanced images are characterized by high numbers of edgels (i.e., pixels belonging to an edge), and that an enhanced image should have a higher intensity of the edges, compared to its non-enhanced counterpart (Saitoh, 1999). Therefore, a good enhancement technique should yield satisfactory results in the case of standard vision processing tasks, such as segmentation or edge detection (Starck et al., 2003). Here, to evaluate image enhancement of MRI data, we employ the method proposed by Canny (1986), which is a highly reliable and mathematically welldefined edge detector. This approach deals with weak edges and accurately determines edgels, by applying a double threshold (to identify potential edges) and a hysteresis-based edge tracking. Let $\mathcal{M}_{\text {Canny }}$ be the edge map yielded by the Canny's edge detector, which is a binary image wherein only edgels are set to 1 . The number of detected edges (\#DE) in $\mathcal{M}_{\text {Canny }}$ is computed as:

$$
\# \mathrm{DE}=\sum_{a=1}^{M} \sum_{b=1}^{N} \mathcal{M}_{\text {Canny }}(a, b)
$$


An additional metrics, called Absolute Mean Brightness Error $(A M B E)$ (Chen \& Ramli, 2003, Arriaga-Garcia et al. 2014), can be employed to measure the brightness preservation of the enhanced image:

$$
A M B E=\frac{\left|\mathbb{E}\left[I_{\text {orig }}\right]-\mathbb{E}\left[\tilde{I}_{\text {enh }}\right]\right|}{L}
$$

where $\mathbb{E}[\cdot]$ denotes the expected (mean) value of a gray level distribution. $A M B E$ is normalized in $[0,1]$, divided by $L=l_{\text {in }}^{(\max )}-l_{\text {in }}^{(\min )}$, which is the dynamic range of the input gray-scale (in our case, $\mathcal{L}^{\prime}{ }_{i n}$ ). Note that low values of $A M B E$ denote that the mean brightness of the original image is preserved.

Finally, we consider an alternative quality metrics called Structural Similarity Index (SSIM) (Wang et al., 2004), used to assess the image degradation perceived as variations in structural information (Bhandari et al., 2016). The structural information defines the attributes that represent the structure of objects in the image, independently of the average luminance and contrast. In particular, local luminance and contrast are taken into account since overall values of luminance and contrast can remarkably vary across the whole image. SSIM is based on the degradation of structural information - assuming that human visual perception is highly adapted for extracting structural information from a scene - and compares local patterns of pixel intensities. As a matter of fact, natural image signals are highly structured, since pixels are strongly dependent on each other, especially those close by. These dependencies convey important information about the structure of the objects in the viewing field. Let $\mathbf{X}$ and $\mathbf{Y}$ be the $I_{\text {orig }}$ and $I_{\text {enh }}$ image signals, respectively; SSIM combines three relatively independent terms:

- the luminance comparison $l(\mathbf{X}, \mathbf{Y})=\frac{2 \mu_{\mathbf{X}} \mu_{\mathbf{Y}}+\kappa_{1}}{\mu_{\mathbf{X}}^{2}+\mu_{\mathbf{Y}}^{2}+\kappa_{1}}$

- the contrast comparison $c(\mathbf{X}, \mathbf{Y})=\frac{2 \sigma_{\mathbf{X}} \sigma_{\mathbf{Y}}+\kappa_{2}}{\sigma_{\mathbf{X}}^{2}+\sigma_{\mathbf{Y}}^{2}+\kappa_{2}}$;

- the structural comparison $s(\mathbf{X}, \mathbf{Y})=\frac{\sigma_{\mathbf{X Y}}+\kappa_{3}}{\sigma_{\mathbf{X}} \sigma_{\mathbf{Y}}+\kappa_{3}}$;

where $\mu_{\mathbf{X}}, \mu_{\mathbf{Y}}, \sigma_{\mathbf{X}}, \sigma_{\mathbf{Y}}$, and $\sigma_{\mathbf{X Y}}$ are the local means, standard deviations, and cross-covariance for the images $\mathbf{X}$ and $\mathbf{Y}$, while $\kappa_{1}, \kappa_{2}, \kappa_{3} \in \mathbb{R}^{+}$are regu- 
larization constants for luminance, contrast, and structural terms, respectively, exploited to avoid instability in the case of image regions characterized by local mean or standard deviation close to zero. Typically, small non-zero values are employed for these constants; according to Wang et al. (2004), an appropriate setting is $\kappa_{1}=(0.01 \cdot L)^{2}, \kappa_{2}=(0.03 \cdot L)^{2}, \kappa_{3}=\kappa_{2} / 2$, where $L$ is the dynamic range of the pixel values in $I_{\text {orig }}$ (represented in $\mathcal{L}^{\prime}{ }_{i n}$ ). SSIM is then computed by combining the components described above:

$$
S S I M=l(\mathbf{X}, \mathbf{Y})^{\alpha} \cdot c(\mathbf{X}, \mathbf{Y})^{\beta} \cdot s(\mathbf{X}, \mathbf{Y})^{\gamma}
$$

where $\alpha, \beta, \gamma>0$ are weighting exponents. As reported in Wang et al. (2004), if $\alpha=\beta=\gamma=1$ and $\kappa_{3}=\kappa_{2} / 2$, the SSIM becomes:

$$
S S I M=\frac{\left(2 \mu_{\mathbf{X}} \mu_{\mathbf{Y}}+\kappa_{1}\right)\left(2 \sigma_{\mathbf{X Y}}+\kappa_{2}\right)}{\left(\mu_{\mathbf{X}}^{2}+\mu_{\mathbf{Y}}^{2}+\kappa_{1}\right)\left(\sigma_{\mathbf{X}}^{2}+\sigma_{\mathbf{Y}}^{2}+\kappa_{2}\right)} .
$$

SSIM generalizes the Universal Quality Index (UQI), defined in Wang \& Bovik (2002), which is obtained by setting $\kappa_{1}=\kappa_{2}=0$, and yields unstable results when either $\left(\mu_{\mathbf{X}}^{2}+\mu_{\mathbf{Y}}^{2}\right)$ or $\left(\sigma_{\mathbf{X}}^{2}+\sigma_{\mathbf{Y}}^{2}\right)$ tends to zero. Notice that the higher the SSIM value, the higher the structural similarity, implying that the enhanced image $I_{e n h}$ and the original image $I_{\text {orig }}$ are quantitatively similar.

\section{Results}

This section presents the experimental results achieved by our image enhancement method. We first analyze the performances of MedGA by varying the parameter settings of the GA at the basis of our methodology; we then compared it against the most common and popular image enhancement techniques in the image processing field (see Gonzalez \& Woods (2002) for additional details).

\subsection{MedGA settings analysis and calibration}

To analyze the performances of MedGA and identify the best settings for the image enhancement problem, we considered a calibration set consisting of 
80 medical images randomly selected from the available MRI data (described in Section 4.1, and we varied the settings of MedGA used throughout the optimization process, that is: $(i)$ the size of the population $|P| \in\{50,100,150,200\}$; (ii) the crossover probability $p_{c} \in\{0.8,0.85,0.9,0.95,1.0\} ;$ (iii) the mutation probability $p_{m} \in\{0.01,0.05,0.1,0.2\} ;(i v)$ the size of the tournament selection strategy $k \in\{5,10,15,20\}$. In all tests MedGA was run for $T=100$ iterations.

Each MedGA execution was performed by varying one setting at a time, for a total of 320 different settings tested and a total number of $320 \times 80=25600$ MedGA executions.

The results of these tests (data not shown) highlighted that, for each value of $|P|$, the best settings in terms of fitness values achieved are:

1. $|P|=50, p_{c}=0.85, p_{m}=0.01, k=15$;

2. $|P|=100, p_{c}=0.9, p_{m}=0.01, k=20$;

3. $|P|=150, p_{c}=0.85, p_{m}=0.01, k=20$;

4. $|P|=200, p_{c}=0.85, p_{m}=0.01, k=20$.

Figure 4 reports the comparison of the performances achieved by MedGA optimization of the same batch of 10 images running a single core of the Intel Xeon E5-2440 CPU with 2.40 GHz clock frequency. On the other hand, by ex- 


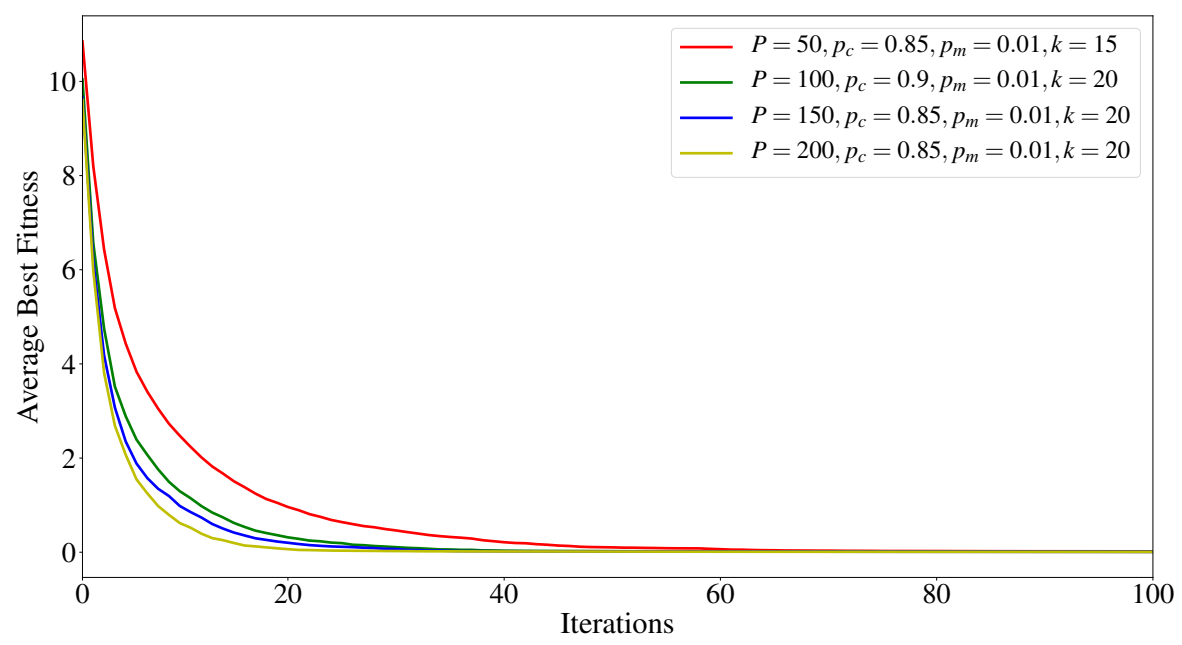

Figure 4: Comparison of the ABF achieved by MedGA with the best parameterizations found for each value of $|P|$ tested here. The average was computed over the results of the optimization of $80 \mathrm{MR}$ images.

ploiting the 6 cores of the same CPU to execute the parallel version of MedGA, we achieved up to $3.6 \times$ speed-up with respect to the sequential version. The results achieved using the parallel version of MedGA confirm the importance of HPC solutions in the field of real healthcare environment to obtain clinically feasible outcomes, that is, enhancing MR images in reasonable time for medical imaging practice.

530

By considering both the performance of MedGA in terms of ABF and the running time required to process 80 images, we selected the parameter settings $|P|=100, p_{c}=0.9, p_{m}=0.01, k=20$ as the best trade-off characterized by a good convergence speed and an adequate running time (for this specific application), and we exploited this configuration for all tests reported and discussed in the following section.

\subsection{Comparison with state-of-the-art methods}

The performances of MedGA were compared against the following image enhancement techniques: 
- Histogram Equalization (HE) (Paranjape, 2009; Hall, 1974), which adjusts pixel intensities for contrast enhancement according to the normalized histogram of the original image $I_{\text {orig. }}$. With $\mathrm{HE}$, gray levels are more uniformly distributed on the histogram, by spreading the most frequent intensity values;

- Bi-Histogram Equalization (Bi-HE) (Kim 1997) - a modification of the traditional $\mathrm{HE}$ - that addresses issues concerning mean brightness preservation;

- Gamma Transformation (GT), which is a non-linear operation using the power-law relationship $s(r)=c r^{\gamma}$, where $r$ and $s$ are the input and the output gray-scale values, respectively, and $c$ is a multiplication constant ( $c=1$ in the following tests). The parameter $\gamma$ is set to values greater than 1 (i.e., decoding gamma) to obtain a gamma expansion, or to values smaller than 1 (i.e., encoding gamma) to realize a gamma compression (see Fig. 5a). In our tests we considered the values $\gamma=0.4$ and $\gamma=2.5$, as higher (lower) values of $\gamma$ tend to logarithmic (anti-logarithmic) functions, resulting in an excessively bright (dark) output image, unsuitable for practical medical applications (Gandhamal et al. 2017);

- Sigmoid intensity Transformation (ST) function (Fig. 5b), also called Sshaped curve, which is a global non-linear mapping defined as follows:

$$
s(r)=\frac{l_{i n}^{(\max )}}{1+\exp (-\lambda(r-\alpha))},
$$

where $l_{\text {in }}^{(\max )}=\max \left\{\mathcal{L}_{i n}\right\}=\max \left\{\mathcal{L}^{\prime}{ }_{\text {in }}\right\}$ is the asymptotic maximum value of the function, $\alpha=\frac{1}{2}\left(l_{i n}^{(\max )}-l_{\text {in }}^{(\min )}\right)$ is the midpoint value, and $\lambda$ defines the function steepness. This transformation stretches the intensity around the level $\alpha$, by making the hypo-intense histogram part darker and the hyper-intense histogram part brighter. Thus, the difference between the minimum and maximum gray values and the gradient magnitude of the image are increased, obtaining strong edges (Gandhamal et al. 2017). 


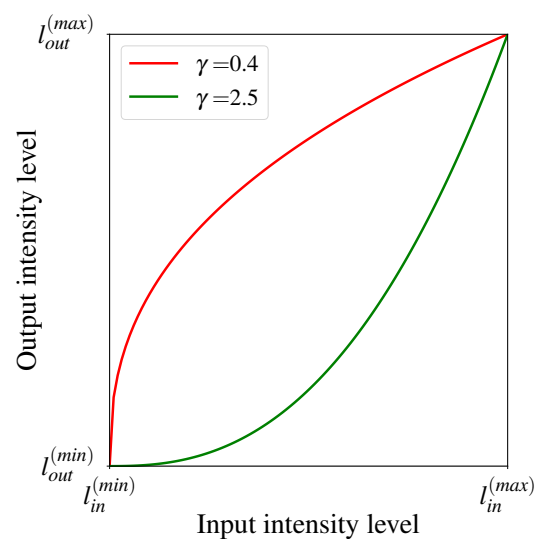

(a)

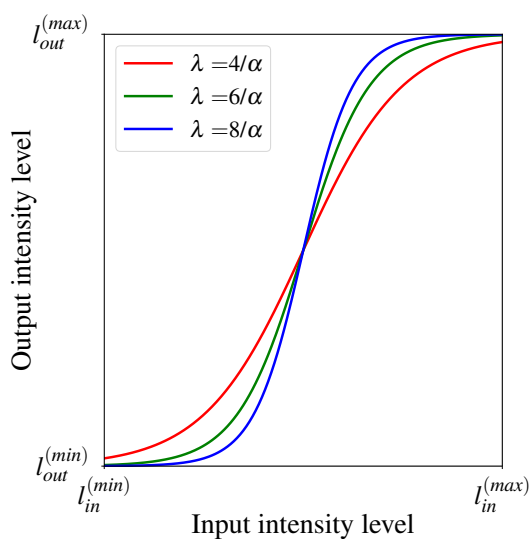

(b)

Figure 5: Plots of the implemented global non-linear intensity transformations for image enhancement: (a) Gamma Transformation; (b) Sigmoid intensity Transformation. We report on the $x$-axis the input intensity range $\left[l_{i n}^{(\min )}, \ldots, l_{i n}^{(\max )}\right]$, and on the $y$-axis the output intensity range $\left[l_{\text {out }}^{(\min )}, \ldots, l_{\text {out }}^{(\max )}\right]$.

In our tests, we used sigmoid functions that allow for considering the entire input dynamic range, by varying the curve slope with the values $\lambda \in\left\{\frac{4}{\alpha}, \frac{6}{\alpha}, \frac{8}{\alpha}\right\}$.

In order to achieve a thorough comparison between MedGA and the enhancement techniques listed above, we exploited the entire set of MRI data consisting in 163 medical images of uterine fibroids (including both calibration and unseen data). For each technique, we computed the metrics PSNR, \#DE, $A M B E, S S I M$ defined in Section 4.2 The resulting values are given in Fig. 6 (boxplots) and in Table 1 (median, mean and standard deviation). Overall, these results highlight that MedGA significantly outperforms the conventional image enhancement approaches in terms of signal quality and perceived structural information in the images, while preserving the input mean brightness. As a matter of fact, MedGA achieves the highest PSNR and SSIM median and mean values (see Fig. 6 and Table 1). Concerning the other enhancement techniques, we observe that Bi-HE achieves better performances with respect to 


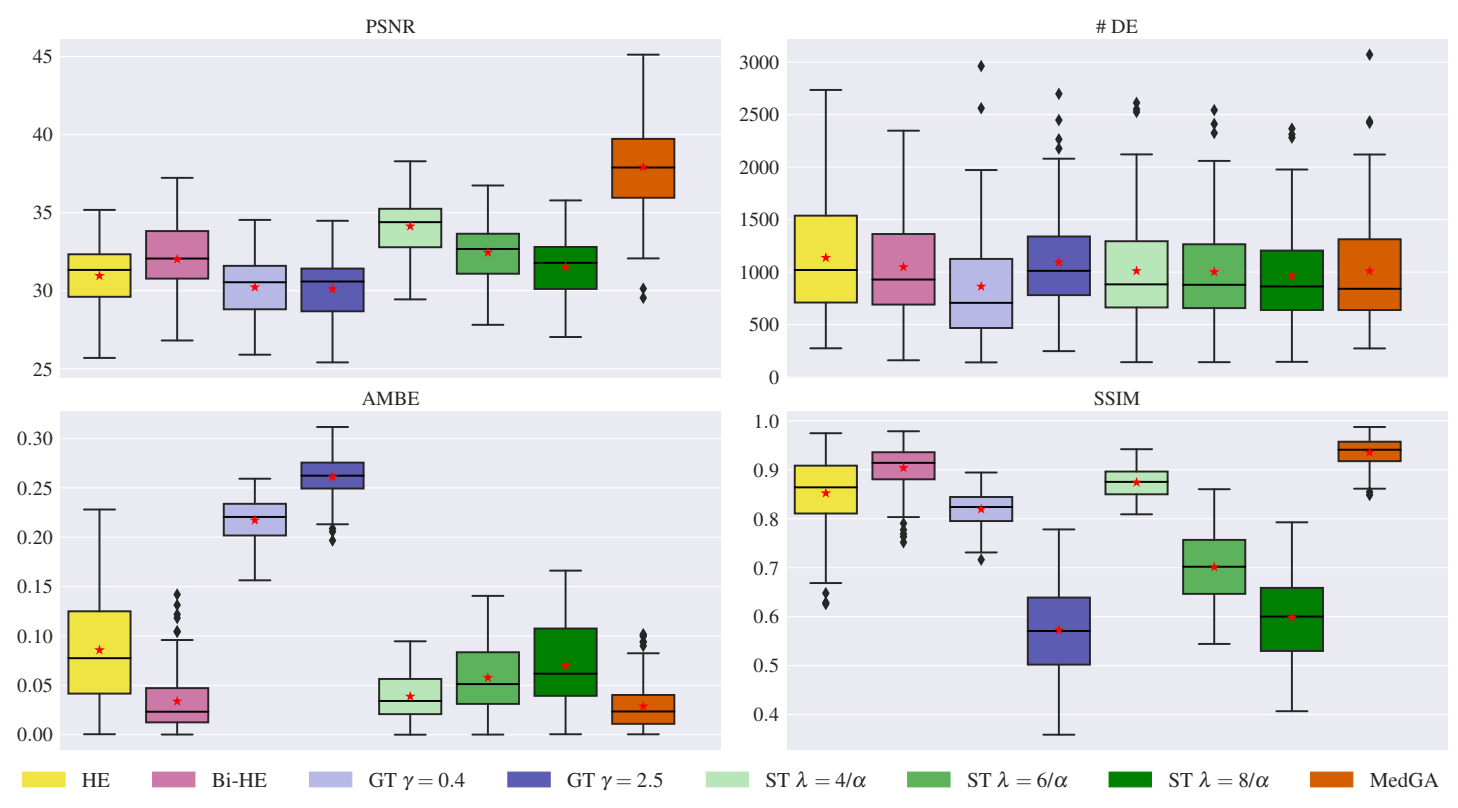

Figure 6: Boxplots of the image enhancement evaluation metrics considered in this work, obtained on an MRI dataset composed of 18 patients with uterine fibroids who had undergone MRgFUS treatment (total number of MR slices: 163). The lower and the upper bounds of each box represent the first and third quartiles of the statistical distribution, respectively, corresponding to the interquartile range. The median and the mean values are represented by a black solid line and a red star, respectively. Whiskers value is 1.5 in all cases and outliers are displayed as black diamonds. 
Table 1: Median, mean and standard deviation of image enhancement evaluation metrics achieved by HE, Bi-HE, GT (with $\gamma \in\{0.4,2.5\}$ ), ST (with $\lambda \in\{4 / \alpha, 6 / \alpha, 8 / \alpha\}$ ) and MedGA, considering the uterine fibroid MRI dataset consisting in a total of 163 medical images.

\begin{tabular}{|c|c|c|c|c|c|c|c|c|c|c|c|c|}
\hline & \multicolumn{3}{|c|}{ PSNR } & \multicolumn{3}{|c|}{$\# \mathbf{D E}$} & \multicolumn{3}{|c|}{ AMBE } & \multicolumn{3}{|c|}{ SSIM } \\
\hline & Median & Mean & Std. Dev. & Median & Mean & Std. Dev. & Median & Mean & Std. Dev. & Median & Mean & Std. Dev \\
\hline $\mathrm{HE}$ & 31.331 & 30.957 & 2.068 & 1020 & 1136.865 & 561.791 & 0.077 & 0.086 & 0.053 & 0.864 & 0.852 & 0.072 \\
\hline $\mathrm{Bi}-\mathrm{HE}$ & 32.059 & 32.014 & 2.232 & 929 & 1048.301 & 472.550 & 0.023 & 0.034 & 0.029 & 0.914 & 0.904 & 0.046 \\
\hline GT $\gamma=0.4$ & 30.540 & 30.223 & 1.994 & 707 & 862.871 & 510.196 & 0.221 & 0.217 & 0.022 & 0.824 & 0.820 & 0.033 \\
\hline $\mathrm{GT} \gamma=2.5$ & 30.588 & 30.106 & 2.063 & 1012 & 1092.847 & 444.502 & 0.262 & 0.261 & 0.021 & 0.571 & 0.573 & 0.094 \\
\hline $\mathrm{ST} \lambda=4 / \alpha$ & 34.389 & 34.127 & 1.880 & 883 & 1011.061 & 468.799 & 0.034 & 0.039 & 0.024 & 0.875 & 0.874 & 0.031 \\
\hline $\mathrm{ST} \lambda=6 / \alpha$ & 32.673 & 32.449 & 1.950 & 879 & 1002.196 & 443.344 & 0.051 & 0.058 & 0.035 & 0.702 & 0.701 & 0.074 \\
\hline $\mathrm{ST} \lambda=8 / \alpha$ & 31.782 & 31.513 & 1.983 & 863 & 962.141 & 411.109 & 0.062 & 0.070 & 0.042 & 0.600 & 0.598 & 0.089 \\
\hline MedGA & 37.891 & 37.914 & 2.821 & 841 & 1008.773 & 499.906 & 0.024 & 0.029 & 0.023 & 0.941 & 0.936 & 0.029 \\
\hline
\end{tabular}

HE, while increasing the values of $\gamma$ and $\lambda$ corresponds to a degradation of the performances of GT and ST, respectively.

For what concerns the \#DE metrics, HE over-enhances the processed MR images, as denoted by the highest median and mean values achieved, while Bi-HE allows for the preservation of the mean brightness, as also indicated by the lowest mean value of $A M B E$. On the contrary, GT achieved the worst performance in terms of $A M B E$ metrics. This poor result is due to the intrinsic nature of GT that transforms the input gray-scale range into a darker $(\gamma>1)$ or brighter $(\gamma<1)$ one, by increasing the number of hyper-intense and hypointense pixels, respectively. By doing so, GT does not preserve the input mean brightness, thus obtaining the highest values of $A M B E$. All the other methods are characterized by comparable \#DE values, while the PSNR is characterized by very high mean values in the case of GT with respect to the other techniques. On the one hand, considering the SSIM metrics, GT with $\gamma=0.4$ remarkably yields better results compared to GT with $\gamma=2.5$, especially in the case of the SSIM; on the other hand, all metrics related to the tested ST functions show that their performances decrease as the value of $\lambda$ increases. This phenomenon is related to the rapid variation characterizing the highest values of $\lambda$, which do not allow for taking into consideration the existing dependency among the pixels, especially those in the neighborhood.

Finally, we show in Fig. 7 two examples of MR images enhanced by using all 

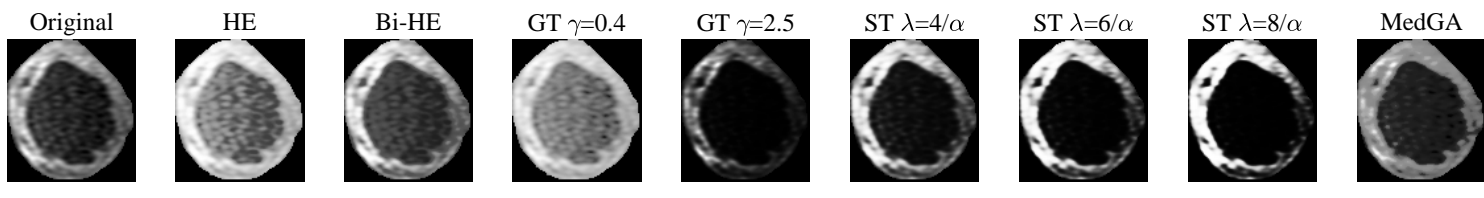

(a)
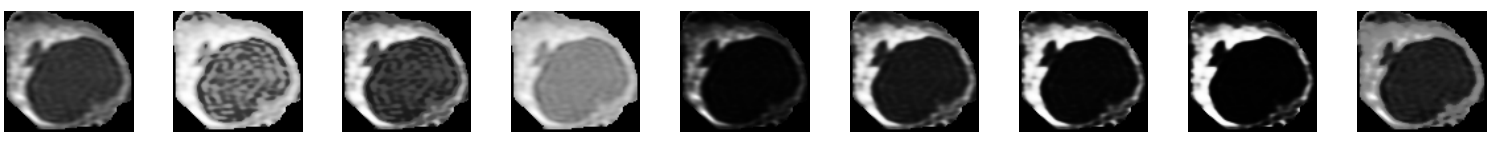

(b)

Figure 7: Examples of enhancement achieved on medical images of uterine fibroids by HE, Bi-HE, GT (with $\gamma \in\{0.4,2.5\}$ ), ST (with $\lambda \in\{4 / \alpha, 6 / \alpha, 8 / \alpha\}$ ) and MedGA, compared with the original input MR image.

the methods considered in this work. As it can be observed, MedGA strengthens the ROI edges by enhancing details and features useful for image binarization; this result confirms, from a qualitative perspective, the quantitative results presented above.

From an overall view of the metrics, we can claim that the approaches obtaining the highest values of the \#DE measure (i.e., HE and GT with $\gamma=2.5$ ) could imply an over-enhancement of the output image, according to the other image quality metrics. This finding is also confirmed by means of a visual inspection of Fig. 7. where the images enhanced using HE and GT with $\gamma=2.5$ present an inadequate appearance for image observation and interpretation. To conclude, MedGA achieves outstanding results in performance evaluation with respect to classic image enhancement techniques.

\section{Discussion}

The main purpose of state-of-the-art methods for image enhancement is the improvement of the contrast level of the whole image, to obtain a "visually ${ }_{615}$ pleasant" result. On the contrary, MedGA is the first enhancement method that explicitly addresses the challenging issues related to the improvement of medical images that are characterized by a nearly bimodal gray level histogram 
distribution. The solution provided by our intelligent image enhancement system can be beneficial to visually assist physicians in interactive decision-making tasks, as well as to improve the final outcome of downstream automated processing pipelines for useful measurements in the clinical practice (Rueckert et al. 2016).

MedGA also deals with the practical problems regarding the interpretability of the results yielded by advanced Machine Learning and Computational Intelligence methods in medicine (Cabitza et al. 2017). Indeed, the final best solution found by MedGA (i.e., the output gray level histogram) and the corresponding enhanced image are understandable by physicians. In addition, the efficient encoding of the individuals - taking inspiration by (Hashemi et al. 2010) - coupled with effective HPC solutions, allows for a clinically feasible computational framework. We designed a specific fitness function to emphasize the two Gaussian distributions composing a bimodal histogram, while the existing approaches based on evolutionary computation or Swarm Intelligence techniques were conceived for a different purpose, i.e., improving the perceived visual information in terms of image contrast. Thanks to this ad hoc fitness function - differently to the GPbased image enhancement method in (Poli \& Cagnoni, 1997), where no fitness function is defined because the user interactively selects the best solution - and the robustness achieved by means of the calibration step for GA's parameter setting, MedGA does not require any user interaction step. Moreover, MedGA differs from GP-based approaches, in which the final generated solution could have large size (Castelli et al., 2014), so heavily affecting the readability and interpretability of the provided solutions. The main impact of this contribution consists in showing how an effective method based on evolutionary computation can outperform the existing methods in medical image enhancement.

In this work, we tested and validated our approach on MR images regarding ${ }_{645}$ MRgFUS therapy for uterine fibroids. More generally, the application of MedGA can be extended also to real-world problems involving the analysis of images characterized by an underlying bimodal histogram, as in the case of bright-field and fluorescence microscopy imaging (Meijering, 2012). 
Considering the achieved results in terms of \#DE (see Table 1), MedGA's

performance could be further improved - in terms of contrast - by integrating a novel component in the fitness function, which explicitly relies on the number of detected edges. Since this additional component would have a different purpose and a different magnitude, a multi-objective optimization method should be taken into account. In particular, MedGA could be extended by means of an effective evolutionary computation approach, such as NSGA-III (Deb \& Jain. 2014), to simultaneously optimize both conflicting objectives, which consist in maximizing the number of edges while minimizing the distance between the optimal threshold and the two normal distributions.

\section{Conclusion and future work}

A novel image enhancement method based on GAs, specifically tailored for medical images characterized by a bimodal histogram, was proposed in this paper. This computational framework, named MedGA, exploits a fitness function that better reveals the two underlying sub-distributions of the gray level intensities, consequently allowing for an improvement in the results achieved by threshold-based algorithms. Unlike the traditional image enhancement techniques that generally improve the contrast level of the whole image, MedGA focuses on MR image sub-regions characterized by a roughly bimodal histogram, making it valuable in clinical contexts involving CE MRI analysis.

We tested and validated our approach on MR images representing uterine fibroids of patients who underwent MRgFUS therapy. MedGA was compared against the most common image enhancement techniques, overall achieving the best performances with respect to both signal and perceived image quality, while preserving, unlike classic HE techniques, the input mean brightness. This novel medical image enhancement technique was therefore shown to be a promising solution, suitable for medical expert systems.

We remark that, although MedGA exploits the same encoding of individuals defined in (Hashemi et al., 2010) and (Draa \& Bouaziz, 2014) we did not compare 
it to other approaches based on evolutionary computation or Swarm Intelligence techniques (see also (Chen et al., 2018)), since they were conceived for a different purpose, that is, improving the perceived visual information of the whole image. As a matter of fact, these approaches explicitly include in the fitness function both the number of edge pixels and the intensity of such pixels, thus achieving high \#DE values that would consistently lead to over-enhanced images.

As a future extension of this work, in the case of large size images (e.g., $1000 \times 1000$ pixels) we plan to use Graphics Processing Units, which represent an enabling technology for real-time radiology applications (Eklund et al., 2013), since the running time of histograms computation can be considerably reduced by using a parallel implementation (Scheuermann \& Hensley, 2007).

In addition, we plan to integrate MedGA as a pre-processing step within an automatic pipeline defined in the context of MR image classification for efficient computer-assisted segmentation using thresholding techniques, such as (Ridler \& Calvard, 1978, Trussell, 1979, Otsu, 1975). Indeed, MR image segmentation is a compelling task in radiology practice, for instance in brain tumor detection and delineation (Sompong \& Wongthanavasu, 2017). Especially, we plan to apply MedGA to metastatic cancer segmentation in neuro-radiosurgery therapy (Leksell, 1949), wherein the enhancement region must be accurately segmented (Militello et al., 2015a; Rundo et al., 2017). In order to make the resulting expert system fully automatic, the segmentation of the ROI bounding region could be performed by robust computational methods that can ensure results repeatability during patients follow-up. In the case of a significant data availability, this step may be performed by means of approaches based on Deep Neural Networks (Rajchl et al., 2017). In these clinical scenarios, MedGA can be suitably integrated in expert systems tailored for MRgFUS treatment evaluation (Rundo et al. 2019), and brain tumor segmentation in neuro-radiosurgery 705 (Meier et al. 2016). 


\section{Acknowledgment}

We thank the Department of Biopathology and Medical Biotechnologies (DIBIMED) at the University of Palermo, Palermo, Italy, for their support and for supplying the images analyzed in this study.

This work was conducted in part using the resources of the Advanced Computing Center for Research and Education at the Vanderbilt University, Nashville, TN, USA.

\section{References}

\section{References}

715 de Araujo, A. F., Constantinou, C. E., \& Tavares, J. M. R. S. (2014). New artificial life model for image enhancement. Expert Syst. Appl., 41, 58925906. doi:10.1016/j.eswa.2014.03.029

Arriaga-Garcia, E. F., Sanchez-Yanez, R. E., \& Garcia-Hernandez, M. G. (2014). Image enhancement using bi-histogram equalization with adaptive sigmoid functions. In Proc. IEEE International Conference on Electronics, Communications and Computers (CONIELECOMP) (pp. 28-34). IEEE. doi:10.1109/CONIELECOMP. 2014.6808563.

Bellon, E. M., Haacke, E. M., Coleman, P. E., Sacco, D. C., Steiger, D. A., \& Gangarosa, R. E. (1986). MR artifacts: a review. Am. J. Roentgenol., 147, 1271-1281. doi 10.2214/ajr.147.6.1271.

Bhandari, A. K., Kumar, A., Chaudhary, S., \& Singh, G. (2016). A novel color image multilevel thresholding based segmentation using nature inspired

a optimization algorithms. Expert Syst. Appl., 63, 112-133. doi 10.1016/j. eswa.2016.06.044

730 Bianco, S., Ciocca, G., \& Schettini, R. (2017). Combination of video change detection algorithms by genetic programming. IEEE Trans. Evol. Comput., 21, 914-928. doi 10.1109/TEVC.2017.2694160. 
Brown, R. W., Cheng, Y. C. N., Haacke, E. M., Thompson, M. R., \& Venkatesan, R. (2014). Magnetic Resonance Imaging: Physical Principles and Sequence Design. John Wiley \& Sons. doi 10.1002/9781118633953

Cabitza, F., Rasoini, R., \& Gensini, G. F. (2017). Unintended consequences

q of machine learning in medicine. J. Am. Med. Assoc., 318, 517-518. doi 10 . 1001/jama.2017.7797.

Canny, J. (1986). A computational approach to edge detection. IEEE Trans.

740 „ Pattern Anal. Mach. Intell., PAMI-8, 679-698. doi:10.1109/TPAMI.1986. 4767851

Carbonaro, A., \& Zingaretti, P. (1999). A comprehensive approach to imagecontrast enhancement. In Proc. International Conference on Image Analysis and Processing (ICIAP) (pp. 241-246). IEEE. doi:10.1109/ICIAP.1999. 797602

Castelli, M., Vanneschi, L., \& Silva, S. (2014). Prediction of the Unified Parkinson's Disease Rating Scale assessment using a genetic programming system with geometric semantic genetic operators. Expert Syst. Appl., 41, 4608-4616. doi:10.1016/j.eswa.2014.01.018.

750 Castelvecchi, D. (2016). Can we open the black box of AI? Nat. News, 538, 20. doi:10.1038/538020a.

Chen, C. M., Chen, C. C., Wu, M. C., Horng, G., Wu, H. C., Hsueh, S. H., \& Ho, H. Y. (2015). Automatic contrast enhancement of brain MR images using hierarchical correlation histogram analysis. Journal Med. Biol. Eng., 35, 724-734. doi:10.1007/s40846-015-0096-6.

Chen, J., Yu, W., Tian, J., Chen, L., \& Zhou, Z. (2018). Image contrast enhancement using an artificial bee colony algorithm. Swarm Evol. Comput., 38, 287-294. doi $10.1016 /$ j.swevo.2017.09.002. 
Chen, S. D., \& Ramli, A. R. (2003). Minimum mean brightness error bihistogram equalization in contrast enhancement. IEEE Trans. Consumer Electron., 49, 1310-1319. doi:10.1109/TCE.2003.1261234.

Dalcín, L., Paz, R., \& Storti, M. (2005). MPI for Python. J. Parallel Distrib. Comput., 65, 1108-1115. doi:10.1016/j.jpdc.2005.03.010.

Deb, K., \& Jain, H. (2014). An evolutionary many-objective optimization algorithm using reference-point-based nondominated sorting approach, Part I: solving problems with box constraints. IEEE Trans. Evol. Computat., 18, 577-601. doi:10.1109/TEVC.2013.2281535.

Draa, A., \& Bouaziz, A. (2014). An artificial bee colony algorithm for image n contrast enhancement. Swarm Evol. Comput., 16, 69-84. doi 10.1016/j. Swevo.2014.01.003.

Eklund, A., Dufort, P., Forsberg, D., \& LaConte, S. M. (2013). Medical image processing on the GPU - Past, present and future. Med. Image Anal., 17, 1073-1094. doi 10.1016/j.media.2013.05.008.

Evans, P. M. (2008). Anatomical imaging for radiotherapy. Phys. Med. Biol., 53, R151. doi $10.1088 / 0031-9155 / 53 / 12 / R 01$.

Gan, H. S., Swee, T. T., Abdul Karim, A. H., Sayuti, K. A., Abdul Kadir, M. R., Tham, W. K., Wong, L. X., Chaudhary, K. T., Ali, J., \& Yupapin, P. P. (2014). Medical image visual appearance improvement using bihistogram Bezier curve contrast enhancement: data from the osteoarthritis initiative. Sci. World J., 2014, 1-13. doi:10.1155/2014/294104

Gandhamal, A., Talbar, S., Gajre, S., Hani, A. F. M., \& Kumar, D. (2017). Local gray level S-curve transformation-a generalized contrast enhancement 口 technique for medical images. Comput. Biol. Med, 83, 120-133. doi 10.1016/ j.compbiomed.2017.03.001.

785 Gonzalez, R. C., \& Woods, R. E. (2002). Digital Image Processing. (3rd ed.). Upper Saddle River, NJ, USA: Prentice Hall Press. 
Gorny, K. R., Woodrum, D. A., Brown, D. L., Henrichsen, T. L., Weaver, A. L., Amrami, K. K., Hangiandreou, N. J., Edmonson, H. A., Bouwsma, E. V., Stewart, E. A., Gostout, B. S., Ehman, D. A., \& Hesley, G. K. (2011). Magnetic resonance-guided focused ultrasound of uterine leiomyomas: review of a 12-month outcome of 130 clinical patients. J. Vasc. Interv. Radiol., 22, 857-864. doi:10.1016/j.jvir.2011.01.458

Hall, E. L. (1974). Almost uniform distributions for computer image enhancement. IEEE Trans. Comput., 100, 207-208. doi:10.1109/T-C.1974.223892

Hashemi, S., Kiani, S., Noroozi, N., \& Moghaddam, M. E. (2010). An image contrast enhancement method based on genetic algorithm. Pattern Recognit. Lett., 31, 1816-1824. doi $10.1016 /$ j.patrec.2009.12.006.

Holland, J. H. (1992). Adaptation in Natural and Artificial Systems: An Introductory Analysis with Applications to Biology, Control and Artificial Intelligence. Cambridge, MA, USA: MIT Press.

Karaboga, D., \& Basturk, B. (2007). A powerful and efficient algorithm for numerical function optimization: artificial bee colony (ABC) algorithm. $J$. Glob. Optim., 39, 459-471. doi:10.1007/s10898-007-9149-x.

Kennedy, J., \& Eberhart, R. (1995). Particle swarm optimization. In Proc. IEEE International Conference on Neural Networks (pp. 1942-1948). volume 4. doi:10.1109/ICNN.1995.488968.

Kim, Y. T. (1997). Contrast enhancement using brightness preserving bia histogram equalization. IEEE Trans. Consumer Electron., 43, 1-8. doi 10. $1109 / 30.580378$

Kohmura, H., \& Wakahara, T. (2006). Determining optimal filters for binarization of degraded characters in color using genetic algorithms. In 18th International Conference on Pattern Recognition (ICPR 2006) (pp. 661-664). IEEE volume 3. doi:10.1109/ICPR.2006.446. 
Koza, J. R. (1992). Genetic Programming: On the Programming of Computers by Means of Natural Selection. (1st ed.). Cambridge, MA, USA: MIT Press.

Krupinski, E. A. (2010). Current perspectives in medical image perception. Atten. Percept. Psychophys., 72, 1205-1217. doi:10.3758/APP.72.5.1205.

Lambin, P., Leijenaar, R. T. H., Deist, T. M., Peerlings, J., de Jong, E. E. C., van Timmeren, J., Sanduleanu, S., Larue, R. T. H. M., Even, A. J. G., Jochems, A. et al. (2017). Radiomics: the bridge between medical imaging a and personalized medicine. Nat. Rev. Clin. Oncol., 14, 749-762. doi 10.1038/ nrclinonc.2017.141.

Leksell, L. (1949). A stereotaxic apparatus for intracerebral surgery. Acta Chir. Scand., 99, 229-233.

825 Meier, R., Knecht, U., Loosli, T., Bauer, S., Slotboom, J., Wiest, R., \& Reyes, M. (2016). Clinical evaluation of a fully-automatic segmentation method for longitudinal brain tumor volumetry. Sci. Rep., 6. doi:10.1038/srep23376.

Meijering, E. (2012). Cell segmentation: 50 years down the road. IEEE Signal Process. Mag., 29, 140-145. doi:10.1109/MSP.2012.2204190.

830 Metcalfe, P., Liney, G. P., Holloway, L., Walker, A., Barton, M., Delaney, G. P., Vinod, S., \& Tome, W. (2013). The potential for an enhanced role for MRI in radiation-therapy treatment planning. Technol. Cancer. Res. Treat., 12, 429-446. doi $10.7785 /$ tcrt.2012.500342.

Militello, C., Rundo, L., \& Gilardi, M. C. (2014). Applications of imaging processing to MRgFUS treatment for fibroids: a review. Transl. Cancer Res., 3, 472-482. doi 10.3978/j.issn.2218-676X.2014.09.06.

Militello, C., Rundo, L., Vitabile, S., Russo, G., Pisciotta, P., Marletta, F., Ippolito, M., D’Arrigo, C., Midiri, M., \& Gilardi, M. C. (2015a). Gamma Knife treatment planning: MR brain tumor segmentation and volume measurement based on unsupervised fuzzy c-means clustering. Int. J. Imaging Syst. Technol., 25, 213-225. doi 10.1002/ima.22139 
Militello, C., Vitabile, S., Rundo, L., Russo, G., Midiri, M., \& Gilardi, M. C. (2015b). A fully automatic 2D segmentation method for uterine fibroid in MRgFUS treatment evaluation. Comput. Biol. Med., 62, 277-292. doi-10. 1016/j.compbiomed.2015.04.030.

Miller, B. L., \& Goldberg, D. E. (1995). Genetic algorithms, tournament selection, and the effects of noise. Complex Syst., 9, 193-212.

Muangkote, N., Sunat, K., \& Chiewchanwattana, S. (2017). R R-cr-IJADE: an efficient differential evolution algorithm for multilevel image thresholding. Expert Syst. Appl., 90, 272-289. doi:10.1016/j.eswa.2017.08.029.

Munteanu, C., \& Rosa, A. (2004). Gray-scale image enhancement as an automatic process driven by evolution. IEEE Trans. Syst. Man Cybern., 34, 1292-1298. doi 10.1109/TSMCB.2003.818533.

Ortiz, A., Górriz, J. M., Ramírez, J., Salas-Gonzalez, D., \& Llamas-Elvira, J. M. 855 (2013). Two fully-unsupervised methods for MR brain image segmentation q using SOM-based strategies. Appl. Soft Comput., 13, 2668-2682. doi 10. $1016 / \mathrm{j}$.asoc. 2012.11 .020 .

Otsu, N. (1975). A threshold selection method from gray-level histograms. IEEE Trans. Syst. Man Cybern., 11, 23-27. doi 10.1109/TSMC.1979.4310076.

Paranjape, R. B. (2009). Chapter 1 - Fundamental Enhancement Techniques. In I. N. Bankman (Ed.), Handbook of Medical Image Processing and Analysis n (pp. 3-18). Burlington, MA, USA: Academic Press. (2nd ed.). doi 10.1016/ B978-012373904-9.50008-8.

Paulinas, M., \& Ušinskas, A. (2007). A survey of genetic algorithms applications 865 for image enhancement and segmentation. Inf. Technol. Control, 36, 278284.

Poli, R., \& Cagnoni, S. (1997). Genetic programming with user-driven selection: experiments on the evolution of algorithms for image enhancement. In 2nd Annual Conference on Genetic Programming (pp. 269-277). 
Rajchl, M., Lee, M. C. H., Oktay, O., Kamnitsas, K., Passerat-Palmbach, J., Bai, W. et al. (2017). DeepCut: object segmentation from bounding box annotations using convolutional neural networks. IEEE Trans. Med. Imaging, 36, 674-683. doi:10.1109/TMI.2016.2621185.

Rangayyan, R. M. (2009). Part I - Enhancement. In I. N. Bankman (Ed.), Handbook of Medical Image Processing and Analysis (pp. 1-2). Burlington, MA, USA: Academic Press. (2nd ed.). doi 10.1016/B978-012373904-9.50008-8

Ridler, T. W., \& Calvard, S. (1978). Picture thresholding using an iterative 口 selection method. IEEE Trans. Syst. Man Cybern., 8, 630-632. doi 10.1109/ TSMC. 1978.4310039.

${ }_{880}$ Rueckert, D., Glocker, B., \& Kainz, B. (2016). Learning clinically useful information from images: past, present and future. Med. Image Anal., 33, 13-18. doi:10.1016/j.media.2016.06.009.

Rundo, L., Militello, C., Tangherloni, A., Russo, G., Lagalla, R., Mauri, G., Gilardi, M. C., \& Vitabile, S. (2019). Computer-assisted approaches for uterine fibroid segmentation in MRgFUS treatments: quantitative evaluation and clinical feasibility analysis. In Quantifying and Processing Biomedical and Behavioral Signals (pp. 229-241). Springer volume 103 of Smart Innovation, Systems and Technologies. doi 10.1007/978-3-319-95095-2_22.

Rundo, L., Stefano, A., Militello, C., Russo, G., Sabini, M. G., D’Arrigo, C., Marletta, F., Ippolito, M., Mauri, G., Vitabile, S., \& Gilardi, M. C. (2017). A fully automatic approach for multimodal PET and MR image segmentation in Gamma Knife treatment planning. Comput. Methods Programs Biomed., 144, 77-96. doi:10.1016/j.cmpb.2017.03.011.

Saitoh, F. (1999). Image contrast enhancement using genetic algorithm. In Proc. IEEE International Conference on Systems, Man, and Cybernetics (SMC) (pp. 899-904). IEEE volume 4. doi:10.1109/ICSMC.1999.812529. 
Scheuermann, T., \& Hensley, J. (2007). Efficient histogram generation using scattering on GPUs. In Proc. Symposium on Interactive 3D Graphics and Games (pp. 33-37). ACM. doi 10.1145/1230100.1230105.

Shanmugavadivu, P., \& Balasubramanian, K. (2014). Particle swarm optimized multi-objective histogram equalization for image enhancement. Opt. Laser Technol., 57, 243-251. doi:10.1016/j.optlastec.2013.07.013

Singh, M., Verma, A., \& Sharma, N. (2017). Bat optimization based neuron model of stochastic resonance for the enhancement of MR images. Biocybern. Biomed. Eng., 37, 124-134. doi 10.1016/j.bbe.2016.10.006

Sompong, C., \& Wongthanavasu, S. (2017). An efficient brain tumor segmentation based on cellular automata and improved tumor-cut algorithm. Expert Syst. Appl., 72, 231-244. doi:10.1016/j.eswa.2016.10.064.

Sourbron, S. P., \& Buckley, D. L. (2013). Classic models for dynamic contrastenhanced MRI. NMR Biomed., 26, 1004-1027. doi 10.1002/nbm.2940.

Starck, J. L., Murtagh, F., Candes, E. J., \& Donoho, D. L. (2003). Gray and color image contrast enhancement by the curvelet transform. IEEE Trans. Image Process., 12, 706-717. doi:10.1109/TIP.2003.813140.

Styner, M., Brechbuhler, C., Széckely, G., \& Gerig, G. (2000). Parametric estimate of intensity inhomogeneities applied to MRI. IEEE Trans. Med. Imaging , 19, 153-165. doi $10.1109 / 42.845174$.

Toennies, K. D. (2017). Guide to Medical Image Analysis. (3rd ed.). London, UK: Springer-Verlag. doi $10.1007 / 978-1-4471-7320-5$.

Trussell, H. J. (1979). Comments on picture thresholding using an iterative 920

selection method. IEEE Trans. Syst. Man Cybern., 9, 311-311. doi 10.1109/ TSMC. 1979.4310204.

Wang, Z., \& Bovik, A. C. (2002). A universal image quality index. IEEE Signal Process. Lett., 9, 81-84. doi:10.1109/97.995823. 
Wang, Z., Bovik, A. C., Sheikh, H. R., \& Simoncelli, E. P. (2004). Image quality assessment: from error visibility to structural similarity. IEEE Trans. Image Proc., 13, 600-612. doi:10.1109/TIP.2003.819861.

Xue, J. H., \& Zhang, Y. J. (2012). Ridler and Calvard's, Kittler and Illingworth's and Otsu's methods for image thresholding. Pattern Recognit. Lett., 33, 793797. doi:10.1016/j.patrec.2011.01.021 